\title{
EUS-guided placement of fiducial markers for stereotactic body radiation therapy in pancreatic cancer: feasibility, security and a new quality score
}

\section{()ㅛ $\odot \ominus$}

\author{
Authors \\ Mariana Figueiredo ${ }^{1}$, Christelle Bouchart ${ }^{2}$, Luigi Moretti ${ }^{2}$, Laura Mans ${ }^{1}$, Jean-Luc Engelholm³ ${ }^{3}$ Maria-Antonietta Bali ${ }^{3}$, \\ Jean-Luc Van Laethem*, ${ }^{*}$, Pierre Eisendrath*,1,4
}

Institutions

1 Department of Gastroenterology, Hepatopancreatology and Digestive Oncology, Erasme University Hospital, Université Libre de Bruxelles, Belgium

2 Department of Radiation-Oncology, Institut Jules Bordet, Université Libre de Bruxelles, Belgium

3 Department of Radiology, Institut Jules Bordet, Université Libre de Bruxelles, Belgium

4 Department of Gastroenterology, Saint-Pierre University Hospital, Université Libre de Bruxelles, Belgium

\section{Bibliography}

Endoscopy International Open 2021; 09: E253-E257

DOI 10.1055/a-1324-2892

ISSN 2364-3722

(c) 2021. The Author(s).

This is an open access article published by Thieme under the terms of the Creative Commons Attribution-NonDerivative-NonCommercial License, permitting copying and reproduction so long as the original work is given appropriate credit. Contents may not be used for commecial purposes, or adapted, remixed, transformed or built upon. (https://creativecommons.org/licenses/by-nc-nd/4.0/)

Georg Thieme Verlag KG, Rüdigerstraße 14,

70469 Stuttgart, Germany

Corresponding author

Mariana Figueiredo Ferreira, MD, Department of

Gastroenterology, Hepatopancreatology and Digestive

Oncology Erasme Hospital, 808 Route de Lennik, 1070

Brussels, Belgium

Fax: +025554802

mariana.figueiredo.ferreira@erasme.ulb.ac.be

\section{ABSTRACT}

Background and study aims In borderline resectable/locally advanced pancreatic ductal adenocarcinoma (PDAC), stereotactic body radiation therapy (SBRT) is an emerging neoadjuvant treatment option. Endoscopic ultrasound (EUS)-guided insertion of fiducial markers being a prerequisite, our aim was to assess its feasibility and safety and also to evaluate its success, from both the endoscopist's and radiotherapist's perspectives.

Patients and methods We prospectively collected data concerning PDAC patients submitted to EUS-guided fiducial placement, from February 2018 to November 2019. Technical success was defined as at least one marker presumed inside the tumor. Quality success was assessed at pre-SBRT computed tomography, accordingly to the number of markers inside or $<1 \mathrm{~cm}$ from the tumor, number of markers at the tumor extremity, their location in different planes, the distance between them, and their distance from the biliary stent (if present). A new quality score was then proposed and high-quality success defined as at least six of 12 points.

Results Thirty-seven patients were enrolled. A total of 97 fiducials were implanted, with a median of three fiducials per patient (0-4). The technical success rate was $92 \%$, with failure of fiducial placement in three patients. Three patients ( $8 \%$ ) had adverse events (fever, mild acute pancreatitis, and biliary stent migration). At pre-SBRT evaluation, two patients' markers had migrated. The high-quality success rate was $62.5 \%$.

Conclusions Our results contribute to demonstrating the feasibility and safety of EUS-guided fiducial placement for SBRT treatment in PDAC. It is hoped that the newly proposed quality score will pave the way for improving fiducial positioning and SBRT delivery.

\section{Introduction}

Pancreatic ductal adenocarcinoma (PDAC) is now the second most common gastrointestinal cancer and the fourth most le-

* These authors contributed equally thal malignancy in the United States, with a 5-year relative sur- 
vival rate not higher than 9\% [1]. In the combined 28 member states of the European Union, it now ranks as the seventh most common form of cancer, with more than 100,000 new cases estimated in both sexes in 2018 , corresponding to $3 \%$ of all new cancer cases [2].

Surgical excision remains the only curative treatment, with an estimated 5-year survival rate of $20 \%$ after resection. However, most patients are diagnosed after progression to a nonresectable disease, with only $15 \%$ to $20 \%$ being potential candidates for curative surgery at presentation [3]. For patients with borderline resectable or locally advanced PDAC, chemotherapy and radiation therapy are increasingly being proposed for therapeutic management, with the purpose of improving curative resectability rates, local control, and overall survival [3]. In this setting, conventional radiation therapy is now challenged by stereotactic body radiotherapy (SBRT). The pancreatic SBRT technique allows delivery of ablative doses in a highly conformational way, particularly at the tumor/vessel interface in few sessions ( 3 to 5 ), while reducing collateral damage to adjacent organs $[4,5]$. It can be easily integrated into a neoadjuvant approach, notably as an attempt to sterilize these tumor/vessels interface regions, contributing to a higher curative (R0) resection rate, as suggested by previous retrospective and phase I/II studies [6]. Insertion of inert radiopaque gold or polymerbased markers in or close to the tumor is a prerequisite for pancreatic SBRT treatments, allowing for precise targeting and real-time tracking of the tumor. Even though fiducials can be placed surgically or percutaneously (under ultrasound/computed tomography [CT] guidance), endoscopic ultrasound (EUS) placement has been established as the preferred route of insertion of fiducial markers in PDAC, given that surgery is a lot more invasive and percutaneous procedures involve other risks such as bleeding or needle tract tumor seeding [7]. The aim of this study was to assess the feasibility and safety of EUS-guided fiducial placement in two academic centers. Moreover, we intended to evaluate both the technical and qualitative success of this technique, from the endoscopist's and radiotherapist's points of view, respectively.

\section{Material and methods}

\section{Study design}

We prospectively collected and analyzed clinical and technical data regarding all the PDAC patients who underwent EUS-guided placement of fiducial markers in two different academic hospitals (Erasme University Hospital and Saint-Pierre University Hospital, Brussels Belgium). All patients had first received four to six cycles of chemotherapy (folfirinox or gemcitabinenab-paclitaxel). SBRT was then performed if there were no signs of disease progression to complete induction treatment. Patients with distant metastases, poor functional status, ongoing infection, current pregnancy, coagulopathy (international normalized ratio $>1.5$ or platelet count $<75,000$ ) or receiving antiplatelet/anticoagulant medications that could not be safely interrupted before the procedure were excluded. Statistical descriptive analyses were performed using SPSS software version 25 (IBM SPSS, New York, United States). The institutional ethics committees of both centers approved the study protocol, which was created in accordance with the ethical guidelines of the 1975 Declaration of Helsinki. Because this was an observational retrospective study, no prior signed consent was obtained. All authors had access to the collected data and approved the final manuscript.

\section{Technical aspects}

The procedures were routinely performed by two experienced endoscopists (>200 EUS examinations [8]), using a linear EUS scope (GFUCT180, Olympus, Hamburg, Germany or EG3870UTK, Pentax, Hamburg, Germany). Patients were under either deep sedation or general anesthesia and they all received antibiotic prophylaxis prior to fiducial insertion. Two types of radio-opaque markers were used: polymer markers (Polymark $0.8 \times 3 \mathrm{~mm}$ ) back-loaded in a regular 19G FNA needle and gold markers delivered with a pre-loaded $22 \mathrm{G}$ dedicated needle (EchoTip Ultra Fiducial Needle, Cook). Fluoroscopy also was used in most cases to monitor the procedure. Relevant technical data were then included in the final procedure report and entered in a prospective registry.

\section{Outcomes}

Technical success was defined as at least one marker presumed to be inside the tumor at the end of the EUS procedure. At preSBRT simulation CT, the number of visible markers and their location (in regard to the tumor and to each other) were assessed by the radiotherapist. After a collaborative meeting between the endoscopic and radiotherapy teams, a quality score based on SBRT prerequisites was proposed, including the following criteria: number of markers inside or $<1 \mathrm{~cm}$ from the tumor, number of markers located in the border of the tumor, their location in different planes, their distance from the biliary stent (if present), and the distance between the fiducials themselves evaluated by the overlap of the different fiducial expanded volumes (FEV). FEV is a volume comprising a radius of $4 \mathrm{~mm}$ around each fiducial marker, calculated and generated at the dosimetric computed tomography (CT) scan.

The score ranged from 0 to 12 points and high-quality success was defined as a score equal or higher than 6/12 points ( $\triangleright$ Table 1 ). A perfect score would imply the insertion of at least three fiducial markers, placed at the tumor's periphery and/or within $1 \mathrm{~cm}$ of the tumor, in different spatial planes, without overlap between them or with the biliary stent (preferably $>8$ $\mathrm{mm}$ apart).

Secondary adverse events (AEs) also were documented. These included bleeding (if blood transfusion and/or local treatment by endoscopy or arterial embolization was required), perforation (with abdominal pain and extra-visceral air visualized at imaging exam), acute pancreatitis (defined accordingly to the revised Atlanta classification [9]), and post-procedural fever $\left(>37.5^{\circ} \mathrm{C}\right)$. 
- Table 1 Fiducial placement quality score.

\begin{tabular}{|l|l|l|}
\hline Question & Answer & Points \\
\hline $\begin{array}{l}\text { Number of markers inside the tumor or } \\
<1 \mathrm{~cm} \text { ? }\end{array}$ & 1 & 1 \\
\cline { 2 - 3 } & 2 & 2 \\
\hline & $\geq 3$ & 3 \\
\hline $\begin{array}{l}\text { Number of markers in the extremity of the } \\
\text { tumor? }\end{array}$ & 0 & 0 \\
\hline & 1 & 1 \\
\hline $\begin{array}{l}\text { Are the markers positioned in different } \\
\text { spatial planes? }\end{array}$ & $\geq 2$ & 2 \\
\hline $\begin{array}{l}\text { Is the distance from the biliary stent } \\
>1 \text { mm? }\end{array}$ & No & 0 \\
\hline Is there an overlap between FEVs? & Yes & 2 \\
\hline & Yes & 0 \\
\hline & No & 3 \\
\hline TOTAL & & $\ldots / 12$ \\
\hline $\begin{array}{l}\text { FEV, fiducial expanded volume (volume comprising a radius of } 4 \mathrm{~mm} \text { around } \\
\text { each fiducial marker). }\end{array}$ & \\
\hline
\end{tabular}

\section{Results}

From February 2018 to November 2019, a total of 37 patients were enrolled ( $\downarrow$ Table 2 ). All of them had initially been treated with four to six cycles of induction chemotherapy. The majority were male $(54.1 \%)$, with a median age of 60 years (IQR 18$)$. The mean tumor size was $25.9 \pm 7.7 \mathrm{~mm}$ and lesions were mostly located at the pancreatic head (70\%). Almost all the lesions were close or invading at least one vessel, with only one showing no vascular involvement (60\% and $35 \%$ had venous and arterial vessels involvement, respectively).

A total of 97 fiducials were implanted, with a median number of three fiducials placed per patient (range 0-4). The technical success rate was $92 \%$, with failure of fiducials placement in three patients, in two cases due to interposing vessels and in one case due to inability to define the tumor limits (probably altered by the induction chemotherapy). At pre-SBRT CT evaluation, however, there were two patients whose markers had migrated and thus were not visualized. In one case, the patient was directly referred to surgery due to the favorable evolution of the contact between the tumor and vessels after chemotherapy. For the second case, due to the persistence of vascular contacts, the radiotherapist used natural markers for guidance (like the main arterial structures and intra-pancreatic calcifications), which significantly increased the difficulty of treatment delivery. Fiducial migration was also observed in five additional patients, but there were always at least two markers still in place.

Of the 32 patients with visible markers at pre-SBRT evaluation, $76 \%$ had at least two markers inside or $<1 \mathrm{~cm}$ from the tumor and in $75 \%$, there was at least one marker located at the border of the tumor. Nevertheless, marker placement in differ-
- Table 2 Population demographics.

\begin{tabular}{|l|l|}
\hline & $\mathrm{n}=37$ \\
\hline Hospital & \\
\hline - CHU Saint-Pierre & $40.5 \%$ \\
\hline - CUB Erasme & $59.5 \%$ \\
\hline Median age (y) & $60(\mathrm{IQR} 18)$ \\
\hline Male patients (\%) & $54.1 \%$ \\
\hline Prior biliary stenting & $47.4 \%$ \\
\hline Previous FNA/FNB & $100 \%$ \\
\hline Mean tumor size (mm) & $25.9(\mathrm{SD} 7.7)$ \\
\hline Tumor location (\%) & \\
\hline - Head & $70.3 \%$ \\
\hline - Neck & $5.4 \%$ \\
\hline - Uncinate process & $10.8 \%$ \\
\hline - Body & $13.5 \%$ \\
\hline Vascular involvement (\%) & \\
\hline - Venous vessels & $60 \%$ \\
\hline - Arterial vessels & $35 \%$ \\
\hline - No vessels involved & $2.7 \%$ \\
\hline FNA, fine-needle aspiration; FNB, fine-needle biopsy. \\
\hline
\end{tabular}

ent spatial planes was achieved in only $56 \%$ of cases and there were $72 \%$ in which at least two markers were positioned too close to each other, with a FEV overlap ( $\triangleright$ Table 3 ). A high-quality success score $(\geq 6 / 12)$ was therefore achieved in $62.5 \%$ of the cases.

$\mathrm{AE}$ were observed in three patients ( $8 \%$ ), with one case of post-procedural fever that resolved spontaneously, one case of mild acute pancreatitis that recovered after conservative medical treatment, and one case of biliary stent migration.

\section{Discussion}

Our study revealed a high technical success rate (92\%) and a low number of AEs (8\%) associated with EUS-guided fiducial placement, confirming the easibility and safety of this endoscopic procedure. This was first demonstrated in 2006 by Pishvaian et al [7], with no complications observed among the six pancreatic cancer patients who underwent the procedure. A more recent systematic review and meta-analysis of studies evaluating the technical aspects, safety, and efficacy of EUS fiducial placement in gastrointestinal malignancies, reporting on 1155 patients, also revealed favorable results [10]. For pancreatic lesions alone, the rate of technical success was $95 \%$, with a migration rate of $2 \%$ to $7 \%$ and a complication rate ranging between $1 \%$ and $13 \%$, as observed in our series. The latter included minor bleeding and duodenal hematoma, mild pancreatitis, 
- Table 3 Technical and quality results.

\begin{tabular}{|c|c|}
\hline & $n=37$ \\
\hline Technical success rate & $92 \%$ (34 patients) \\
\hline $\begin{array}{l}\text { Median number of inserted markers } \\
\text { (per patient) }\end{array}$ & $3(0-4)$ \\
\hline Adverse events (\%) & $8 \%$ \\
\hline Fever & $2.7 \%$ \\
\hline Mild acute pancreatitis & $2.7 \%$ \\
\hline \multirow[t]{2}{*}{ Biliary stent migration } & $2.7 \%$ \\
\hline & $n=34$ \\
\hline SBRT delivered (\%) & $97.1 \%$ \\
\hline \multirow[t]{2}{*}{ Markers migration rate (\%) } & $5.9 \%$ (2 patients) \\
\hline & $n=32$ \\
\hline \multicolumn{2}{|l|}{ Markers inside the tumor or $<1 \mathrm{~cm}$} \\
\hline - $1(1)$ & $12.5 \%$ \\
\hline - $2(2)$ & $31.3 \%$ \\
\hline - $\geq 3(3)$ & $56.2 \%$ \\
\hline \multicolumn{2}{|l|}{ Markers in the extremity of the tumor } \\
\hline - $0(0)$ & $25 \%$ \\
\hline - $1(1)$ & $68.8 \%$ \\
\hline - $\geq 2(2)$ & $12.5 \%$ \\
\hline \multicolumn{2}{|l|}{ Markers in different planes } \\
\hline - Yes (2) & $56.3 \%$ \\
\hline - No $(0)$ & $43.7 \%$ \\
\hline \multicolumn{2}{|l|}{ Distance from the biliary stent > $1 \mathrm{~mm}$} \\
\hline - No $(0)$ & $15.6 \%$ \\
\hline - Yes (2) & $84.4 \%$ \\
\hline \multicolumn{2}{|l|}{ Overlap FEV } \\
\hline - Yes $(0)$ & $71.9 \%$ \\
\hline - No (3) & $28.1 \%$ \\
\hline
\end{tabular}

(n) = Points attributed accordingly to the quality score.

and cholangitis, and there was no registry of any deaths nor lifethreatening complications [10].

Regarding antibiotic prophylaxis, most of the previous studies evaluating the safety of EUS-guided fiducial placement in pancreatobiliary lesions described its routine use in all cases and reported no infectious AEs. However, in a recently published retrospective study by Chandnani et al, $14.4 \%$ of patients $(n=355)$ did not receive antibiotic prophylaxis. Even though infectious complications were observed, they remained extremely rare, with only three cases of post-EUS infection (one of 51 patients $(2 \%)$ in patients who have not received antibiotics developing acute cholangitis, whereas two of 304 (0.7\%) who had received prophylactic antibiotics developed bacteremia and septic shock, respectively) [11]. To date, there are still no clear guidelines on use of antibiotic prophylaxis in EUS-guided fiducial placement. However, infections seem to be extremely rare, making us consider abandoning routine use of antibiotics in favor of a more selective strategy in the future.

In all of the previously published studies, "technical success" was defined as the ability to place fiducials in the tumor. However, that may be insufficient to allow for appropriate fiducial tracing and thus, to allow for SBRT to be performed. Our study stands out as the first work that proposes a new definition of "quality success" with more recent criteria for optimal fiducial placement for SBRT delivery in pancreatic cancer, in the form of a quality score described in collaboration with an experienced radiotherapist.

The notion of "ideal fiducial geometry" (IFG) was proposed in 2003, with a few recommendations for correctly place the markers, including placing at least three fiducials with a minimum interfiducial distance $>2 \mathrm{~cm}$, a minimum interfiducial angle of $>15$ degrees, and noncollinear placement in the imaging plane [12]. Since then, there have been major technical improvements and changes in radiotherapy that have led to modern SBRT techniques, such as modern planning methods (intensity modulated radiotherapy/volumetric modulated arc therapy), four-dimensional CT (to assess the amplitude and direction of tumor movement during the respiratory cycle), abdominal compression and breath-hold techniques (to restrain tumor movement) and the use of on-board cone-beam CT (for daily tumor position verification) and tracking. Yet to our knowledge, the IFG standards have never been revised and newer guiding criteria have yet to be proposed. Also, to date, there are still no solid data about the impact of the fiducial placement based on the IFG criteria on final SBRT quality.

With our new quality score, optimal marker placement can be achieved with the introduction of at least three markers, ideally inserted at the borders of the tumor, in different spatial planes and with a minimum distance of $1 \mathrm{~cm}$ between them. This allows for triangulation and rigorous tumor contouring in multiple planes, increasing treatment precision and contributing to effective delivery of stereotaxic radiotherapy in terms of the ease and security of treatment administration. Insertion of only one marker or multiple markers placed more than $1 \mathrm{~cm}$ away from the tumor should be avoided, as this would greatly decrease the accuracy of radiation delivery (owing reduction in radiation dose). Finally, because each marker is surrounded by an area of $4 \mathrm{~mm}$ (FEV), which serves as a reference during radiotherapy treatment planning, with the overlap between the different FEVs leading to imaging artifacts, an interfiducial distance of at least $1 \mathrm{~cm}$ is preferable. The same principle applies to the distance between the markers and a biliary stent, if present. Moreover, the latter cannot be used as a marker on its own due to how much it moves during respiration, correlating poorly with tumor localization [13].

When compared to the IFG, our score's criteria seem better adapted to current SBRT practice and also more achievable with EUS-guided fiducial insertion, with a high-quality success rate of $62.5 \%$ in our cohort. It is an easily reproductible score with a potentially significant clinical impact, as it may facilitate 
smoother and more precise delivery of SBRT. However, a direct correlation between optimal fiducial placement and overall oncological outcome remains to be demonstrated, as this is only one of multiple factors influencing the final clinical outcome.

The main limitation of our study was the small number of patients included, which could contribute to underestimating the percentage of fiducial introduction failure or AEs. Information concerning the type of needle employed (19 vs 22 gauge) also is lacking, even though previous studies have suggested that there is no statistical difference between them when it comes to fiducial marker insertion [14]. Also, even though our quality results seem promising, a multicenter prospective study is still needed to validate our quality score.

\section{Conclusions}

In conclusion, our results contribute to demonstrating the feasibility and security of EUS-guided fiducial marker placement for SBRT in PDAC, with encouraging results in regards to technical success and rates of AEs. It is hoped that the newly proposed quality score will lead the way to improvement in fiducial positioning, allowing for optimization of SBRT treatment delivery with better final results.

\section{Competing interests}

The authors declare that they have no conflict of interest.

\section{References}

[1] Siegel RL, Miller KD, Jemal A. Cancer statistics, 2019. CA Cancer J Clin 2019; 69: 7-34

[2] Maisonneuve P. Epidemiology and burden of pancreatic cancer. Presse Médicale 2019; 48: e113-e123

[3] Ducreux M, Cuhna AS, Caramella C et al. Cancer of the pancreas: ESMO Clinical Practice Guidelines for diagnosis, treatment and followup. Ann Oncol 2015; 26: v56-v68
[4] Moningi S, Marciscano AE, Rosati LM et al. Stereotactic body radiation therapy in pancreatic cancer: the new frontier. Expert Rev Anticancer Ther 2014; 14: 1461-1475

[5] Herman JM, Chang DT, Goodman KA et al. Phase 2 multi-institutional trial evaluating gemcitabine and stereotactic body radiotherapy for patients with locally advanced unresectable pancreatic adenocarcinoma: SBRT for Unresectable Pancreatic Cancer. Cancer 2015; 121: 1128-1137

[6] Bouchart C, Navez J, Closset J et al. Novel strategies using modern radiotherapy to improve pancreatic cancer outcomes: toward a new standard? Ther Adv Med Oncol 2020; 12: doi:10.1177/1758835920 936093

[7] Pishvaian AC, Collins B, Gagnon G et al. EUS-guided fiducial placement for CyberKnife radiotherapy of mediastinal and abdominal malignancies. Gastrointest Endosc 2006; 64: 412-417

[8] Wani S, Han S, Simon V et al. Setting minimum standards for training in EUS and ERCP: results from a prospective multicenter study evaluating learning curves and competence among advanced endoscopy trainees. Gastrointest Endosc 2019; 89: 1160-1168.e9

[9] Banks PA, Bollen TL, Dervenis C et al. Classification of acute pancreatitis-2012: revision of the Atlanta classification and definitions by international consensus. Gut 2013; 62: 102-111

[10] Coronel E, Cazacu IM, Sakuraba A et al. EUS-guided fiducial placement for $\mathrm{Gl}$ malignancies: a systematic review and meta-analysis. Gastrointest Endosc 2019; 89: 659-670.e18

[11] Chandnani M, Faisal MF, Glissen-Brown J et al. EUS-guided fiducial placement for pancreatobiliary malignancies: safety, infection risk, and use of peri-procedural antibiotics. Endosc Int Open 2020; 08: E179-E185

[12] Olender D. Fiducials for target localization. CyberKnife Radiosurgery Pract. Guide.Heilbrun MP. Sunnyvale (CA): CyberKnife Society; 2003

[13] Goldstein SD, Ford EC, Duhon M et al. Use of respiratory-correlated four-dimensional computed tomography to determine acceptable treatment margins for locally advanced pancreatic adenocarcinoma. Int J Radiat Oncol 2010; 76: 597-602

[14] Dhadham G, Hoffe S, Harris C et al. Endoscopic ultrasound-guided fiducial marker placement for image-guided radiation therapy without fluoroscopy: safety and technical feasibility. Endosc Int Open 2016; 04: E378-E382 